\title{
2 Population and vital statistics
}

\subsection{Mid-year estimates of resident population}

Thousands

\begin{tabular}{|c|c|c|c|c|c|c|c|c|c|c|c|c|}
\hline & \multicolumn{3}{|c|}{ England and Wales ${ }^{1}$} & \multicolumn{3}{|c|}{ Scotland } & \multicolumn{3}{|c|}{ Northern Ireland } & \multicolumn{3}{|c|}{ United Kingdom ${ }^{1}$} \\
\hline & Males & Females & Persons & Males & Females & Persons & Males & Females & Persons & Males & Females & Persons \\
\hline & BBAE & BBAF & BBAD & BBAH & BBAI & BBAG & BBAK & BBAL & BBAJ & BBAB & BBAC & DYAY \\
\hline 1986 & 24311 & 25687 & 49999 & 2462 & 2649 & 5112 & 768 & 805 & 1574 & 27542 & 29142 & 56684 \\
\hline 1987 & 24371 & 25752 & 50123 & 2455 & 2644 & 5099 & 773 & 809 & 1582 & 27599 & 29205 & 56804 \\
\hline 1988 & 24434 & 25820 & 50254 & 2444 & 2633 & 5077 & 774 & 812 & 1585 & 27652 & 29265 & 56916 \\
\hline 1989 & 24510 & 25898 & 50408 & 2443 & 2635 & 5078 & 776 & 814 & 1590 & 27729 & 29348 & 57076 \\
\hline 1990 & 24597 & 25964 & 50561 & 2444 & 2637 & 5081 & 778 & 818 & 1596 & 27819 & 29419 & 57237 \\
\hline 1991 & 24681 & 26067 & 50748 & 2445 & 2639 & 5083 & 783 & 824 & 1607 & 27909 & 29530 & 57439 \\
\hline 1992 & 24739 & 26136 & 50876 & 2445 & 2640 & 5086 & 792 & 831 & 1623 & 27977 & 29608 & 57585 \\
\hline 1993 & 24793 & 26193 & 50986 & 2448 & 2644 & 5092 & 798 & 837 & 1636 & 28039 & 29675 & 57714 \\
\hline 1994 & 24853 & 26263 & 51116 & 2453 & 2649 & 5102 & 802 & 842 & 1644 & 28108 & 29754 & 57862 \\
\hline 1995 & 24946 & 26326 & 51272 & 2453 & 2650 & 5104 & 804 & 845 & 1649 & 28204 & 29821 & 58025 \\
\hline 1996 & 25030 & 26381 & 51410 & 2447 & 2645 & 5092 & 810 & 851 & 1662 & 28287 & 29877 & 58164 \\
\hline 1997 & 25113 & 26446 & 51560 & 2442 & 2641 & 5083 & 816 & 856 & 1671 & 28371 & 29943 & 58314 \\
\hline 1998 & 25201 & 26519 & 51720 & 2439 & 2638 & 5077 & 819 & 859 & 1678 & 28458 & 30017 & 58475 \\
\hline 2000 & 25438 & 26702 & 52140 & 2432 & 2631 & 5063 & 820 & 862 & 1683 & 28690 & 30196 & 58886 \\
\hline 2001 & 25574 & 26786 & 52360 & 2434 & 2630 & 5064 & 824 & 865 & 1689 & 28832 & 30281 & 59113 \\
\hline 2002 & 25704 & 26868 & 52572 & 2432 & 2623 & 5055 & 829 & 868 & 1697 & 28964 & 30359 & 59323 \\
\hline 2003 & 25841 & 26956 & 52797 & 2435 & 2623 & 5057 & 833 & 870 & 1703 & 29109 & 30449 & 59557 \\
\hline 2004 & 25995 & 27062 & 53057 & 2446 & 2632 & 5078 & 836 & 874 & 1710 & 29278 & 30568 & 59846 \\
\hline 2005 & 26197 & 27223 & 53419 & 2456 & 2639 & 5095 & 844 & 880 & 1724 & 29497 & 30741 & 60238 \\
\hline 2006 & 26371 & 27358 & 53729 & 2469 & 2647 & 5117 & 853 & 888 & 1742 & 29694 & 30893 & 60587 \\
\hline 2007 & 26569 & 27503 & 54072 & 2486 & 2659 & 5144 & 862 & 897 & 1759 & 29916 & 31059 & 60975 \\
\hline
\end{tabular}

1 Data for 2002-2005 for England and Wales and United Kingdom has been revised due to improved International Migration.

Sources: Office for National Statistics General Register Office (Scotland) Northern Ireland Statistics and Research Agency

\subsection{Age distribution of estimated resident population at 30 June 2007}

Thousands

\begin{tabular}{|c|c|c|c|c|c|c|c|c|c|c|c|}
\hline & \multicolumn{11}{|c|}{ Resident population } \\
\hline & \multicolumn{2}{|c|}{ England and Wales ${ }^{1}$} & \multicolumn{2}{|c|}{ Wales } & \multicolumn{2}{|c|}{ Scotland } & \multicolumn{2}{|c|}{ Northern Ireland } & \multicolumn{3}{|c|}{ United Kingdom ${ }^{1}$} \\
\hline & Males & Females & Males & Females & Males & Females & Males & Females & Males & Females & Persons \\
\hline $0-4$ & 1641 & 1562 & 84 & 80 & 141 & 134 & 59 & 56 & 1841 & 1752 & 3593 \\
\hline $5-9$ & 1552 & 1484 & 85 & 81 & 140 & 134 & 59 & 56 & 1750 & 1674 & 3424 \\
\hline $10-14$ & 1680 & 1598 & 96 & 91 & 155 & 148 & 63 & 60 & 1898 & 1806 & 3704 \\
\hline $15-19$ & 1834 & 1724 & 105 & 99 & 169 & 160 & 66 & 62 & 2069 & 1947 & 4016 \\
\hline $20-24$ & 1881 & 1780 & 104 & 99 & 175 & 172 & 68 & 64 & 2124 & 2016 & 4141 \\
\hline $25-29$ & 1770 & 1756 & 84 & 84 & 164 & 160 & 58 & 58 & 1992 & 1974 & 3966 \\
\hline $30-34$ & 1738 & 1735 & 79 & 82 & 149 & 156 & 57 & 58 & 1944 & 1949 & 3893 \\
\hline $35-39$ & 2003 & 2028 & 97 & 104 & 180 & 195 & 63 & 65 & 2246 & 2288 & 4534 \\
\hline $40-44$ & 2073 & 2105 & 106 & 111 & 195 & 211 & 64 & 67 & 2332 & 2382 & 4714 \\
\hline $45-49$ & 1853 & 1891 & 99 & 104 & 187 & 199 & 59 & 62 & 2099 & 2151 & 4250 \\
\hline $50-54$ & 1622 & 1660 & 92 & 96 & 168 & 175 & 52 & 52 & 1842 & 1888 & 3730 \\
\hline $55-59$ & 1635 & 1686 & 98 & 101 & 162 & 169 & 47 & 49 & 1845 & 1903 & 3748 \\
\hline $65-69$ & 1148 & 1234 & 72 & 77 & 114 & 130 & 34 & 37 & 1296 & 1401 & 2697 \\
\hline $70-74$ & 979 & 1108 & 60 & 67 & 96 & 118 & 28 & 33 & 1102 & 1259 & 2360 \\
\hline $75-79$ & 769 & 982 & 46 & 58 & 71 & 100 & 20 & 29 & 861 & 1111 & 1972 \\
\hline $80-84$ & 513 & 785 & 32 & 49 & 44 & 74 & 13 & 22 & 570 & 881 & 1452 \\
\hline $85-89$ & 268 & 520 & 16 & 32 & 21 & 45 & 6 & 13 & 295 & 578 & 873 \\
\hline 90 and over & 99 & 286 & 6 & 16 & 8 & 24 & 2 & 6 & 108 & 317 & 425 \\
\hline $0-14$ & 4872 & 4644 & 265 & 251 & 436 & 415 & 182 & 172 & 5490 & 5231 & 10721 \\
\hline $15-64$ & 17921 & 17945 & 957 & 975 & 1696 & 1752 & 577 & 585 & 20194 & 20281 & 40475 \\
\hline 65 and over & 3775 & 4915 & 231 & 299 & 354 & 491 & 103 & 140 & 4233 & 5546 & 9779 \\
\hline All ages & 26569 & 27503 & 1454 & 1526 & 2486 & 2659 & 862 & 897 & 29916 & 31059 & 60975 \\
\hline
\end{tabular}




\subsection{Births $^{1}$ and marriages}

Thousands

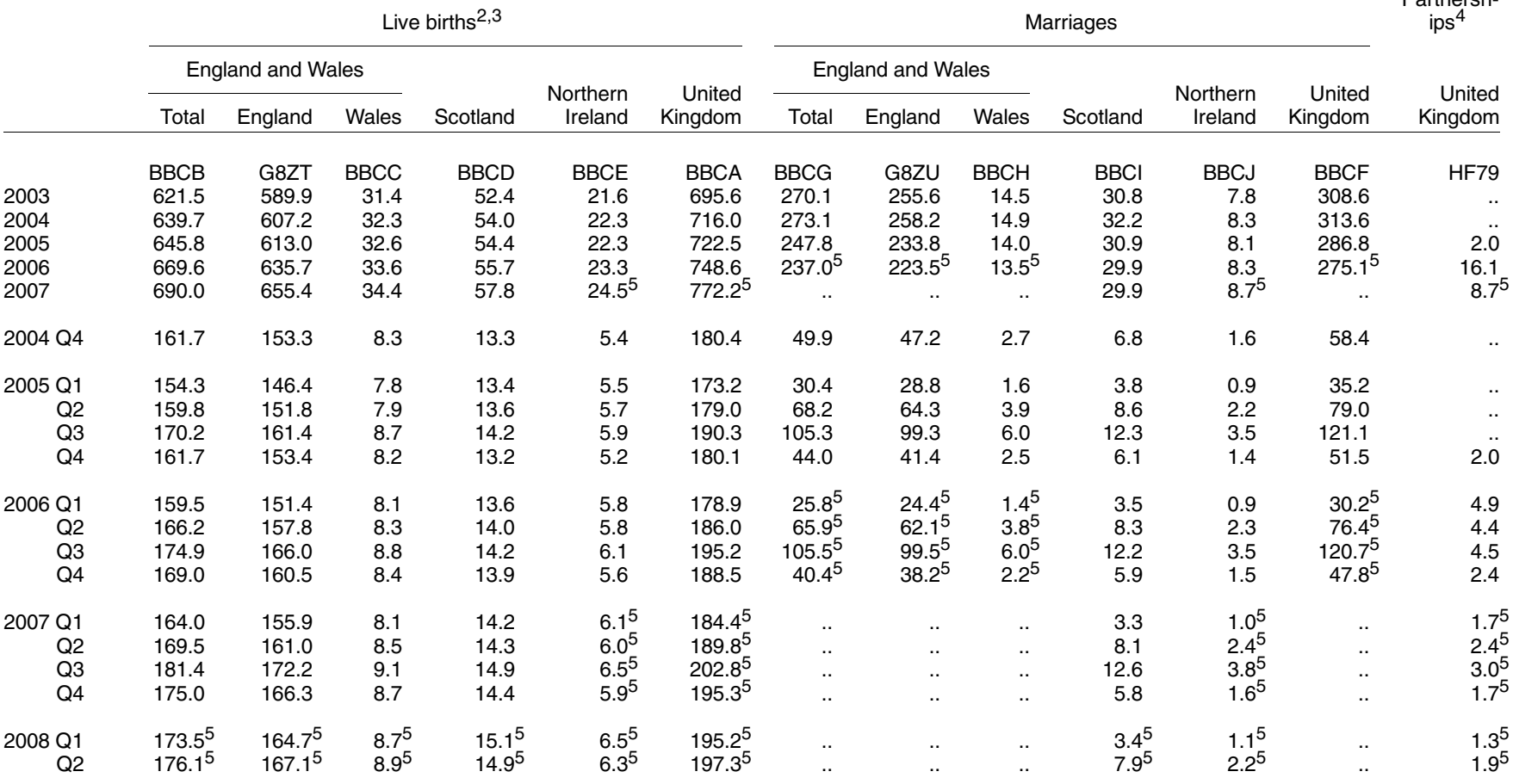

Note: Figures may not add exactly due to rounding.

1 Excluding stillbirths.

2 Birth figures for England and also for Wales each exclude events

for persons usually resident outside England and Wales. These event

are however, included in the totals for England and Wales combined, and

for the United Kingdom.
3 For England and Wales, figures relate to numbers occurring in a period; for Scotland and Northern Ireland, figures relate to those registered in a period.

4 Civil Partnerships were made legal on 5th December 2005.

5 Provisional

Sources: Office for National Statistics General Register Office for Scotland; Northern Ireland Statistics \& Research Agency.

\subsection{Deaths registered}

Thousands

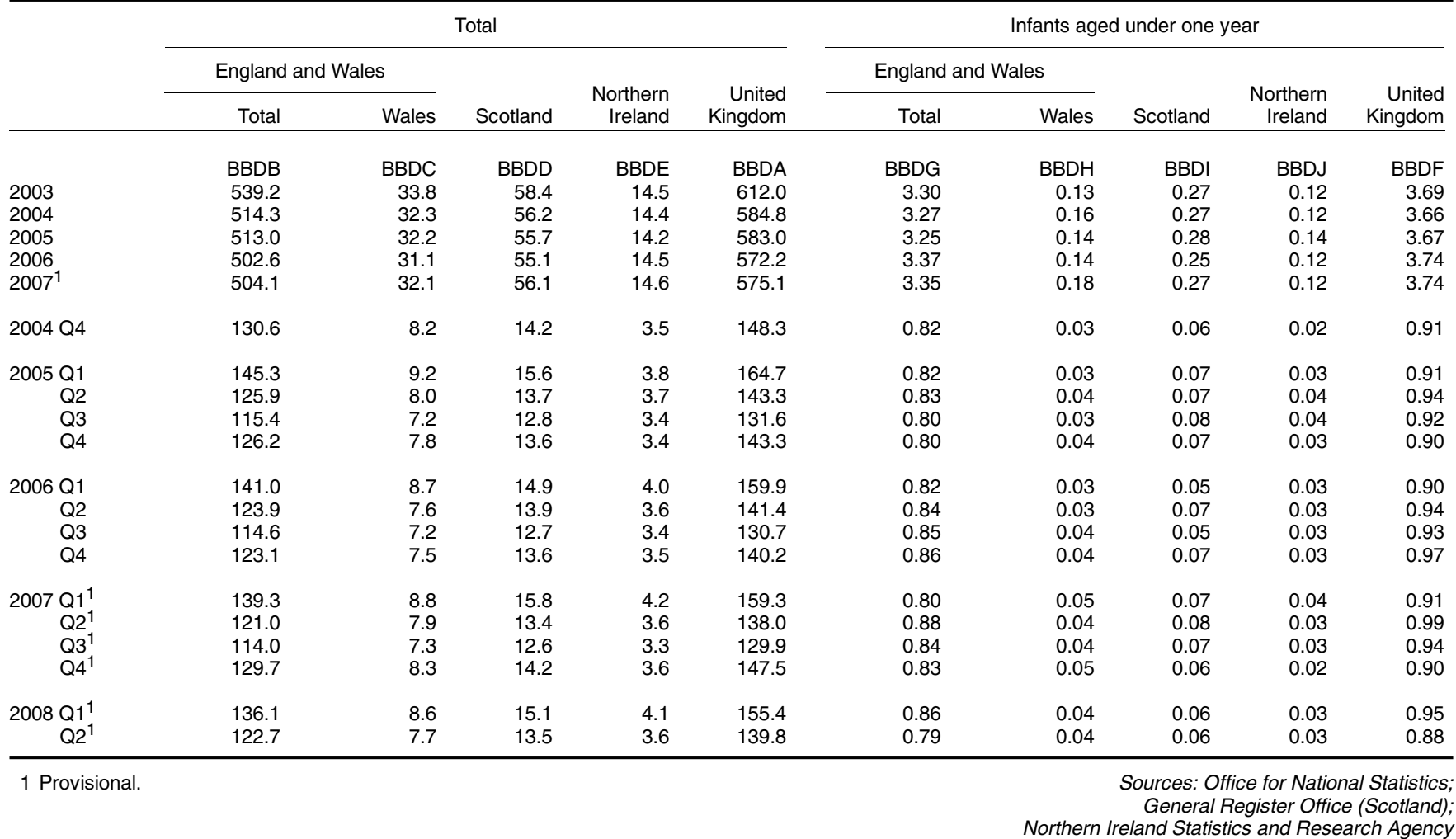

